\title{
Role of Stem Cells and Extracellular Matrix in the Regeneration of Skeletal Muscle
}

\author{
Andrew Dunn, Madison Marcinczyk, \\ Muhamed Talovic, Krishna Patel, Gabriel Haas and \\ Koyal Garg
}

Additional information is available at the end of the chapter

http://dx.doi.org/10.5772/intechopen.75828

\begin{abstract}
Adult skeletal muscle has a remarkable capacity to initiate a rapid and extensive repair process after damage due to injury or degenerative disease. Although satellite cells are the primary skeletal muscle stem cells, there are many reports of non-satellite cell populations with myogenic capacity resident within skeletal muscle. The activity of muscle-resident stem cells during the regeneration process is tightly controlled through the dynamic interactions between intrinsic factors within the cells and extrinsic factors constituting the muscle stem cell niche. The extracellular matrix (ECM) in skeletal muscle plays an integral role in force transmission, structural maintenance, and regulation of stem cell niche. ECM interacts with stem cells either directly by binding cell surface receptors or indirectly through growth factor presentation, and maintains a balance between their quiescence, self-renewal, and differentiation. These interactions are reciprocal since the stem cells can remodel the niche and secrete or degrade ECM components. Natural ECM scaffolds, derived from decellularized tissues can influence stem cell activity both in vitro and in vivo and are widely being investigated for skeletal muscle repair. In this chapter, we discuss the regenerative potential of stem cell populations and ECM bioscaffolds in the treatment of skeletal muscle injury and disease.
\end{abstract}

Keywords: stem cells, extracellular matrix, regeneration, trauma, decellularization

\section{Introduction}

Skeletal muscle injuries are common in military service members and professional athletes and can range from minor sprains and contusions to severe lacerations and penetrating trauma. 
The type and severity of muscle injury greatly influences the healing outcome. While skeletal muscle can regenerate very well following minor injuries, severe penetrating trauma involving physical loss of muscle tissue is well beyond the innate capacity for repair [1, 2]. In acute and self-healing muscle injuries, the typical muscle repair process consists of the destruction and inflammatory phase (1-3 days), the repair phase (3-4 weeks), and the remodeling phase (3-6 months). Extracellular matrix (ECM) deposition can be observed within a week post-injury and is primarily due to the activity of fibroblasts in response to locally produced mediators such as transforming growth factor beta 1 (TGF- $\beta 1$ ) [3]. In chronic muscle injuries the inflammatory phase persists for weeks and the deposition of ECM proceeds more rapidly than myogenesis $[4,5]$. A major impediment to the complete recovery of skeletal muscle postinjury is the development of fibrosis, defined as an abnormal and chronic over proliferation of ECM components [6]. If unresolved, fibrotic tissue can interfere with stem cell activity and myofiber regeneration. Furthermore, since fibrotic tissue lacks the elasticity and contractile properties of native skeletal muscle, patients with muscle fibrosis are dramatically more likely to suffer subsequent muscle injuries, each worsening muscle damage and fibrosis [7]. Fibrotic tissue deposition in the absence of muscle regeneration reduces both muscle strength and range of motion, impeding physical rehabilitation.

In skeletal muscle, fibrosis is also associated with muscular dystrophies. These degenerative diseases are characterized by muscle inflammation and wasting, which compromises patient mobility [1]. Duchenne muscular dystrophy (DMD) is a severe and progressive form of muscular dystrophy that may cause premature death due to respiratory and cardiac failure [8]. Despite continuing efforts, there are currently no effective therapies for severe muscle trauma or DMD. Recently, many novel therapeutic strategies focusing on muscle stem cells and ECM have emerged, as discussed below, and their efficacy is under evaluation in both preclinical and clinical studies.

\section{Stem cells for skeletal muscle regeneration}

The skeletal muscle microenvironment is heterogeneous, with diverse cell populations that can be influenced by local structural and biochemical cues. Skeletal muscle is endowed with a remarkable capacity for regeneration, primarily due to the reserve pool of muscle-resident satellite cells. The anatomic location of satellite cells is in proximity to vasculature where they interact with other muscle-resident stem/stromal cells such as mesenchymal stem cells (MSCs) and pericytes through paracrine mechanisms (Figure 1) [9, 10]. A variety of other stem cell populations have also been identified in skeletal muscle including, side population cells [11, 12], fibro/adipogenic progenitors [13, 14] and interstitial stem cells [15]. These cell types share many features with MSCs such as multipotency and cell surface marker expression and are known to undergo proliferation in response to muscle injury [16]. Additionally, muscle-resident or marrow-derived hematopoietic stem cells (HSCs) are known to rapidly colonize skeletal muscle post-injury [9]. Here, we review the contribution of satellite and nonsatellite stem cells to muscle repair in animal models of injury and disease. 


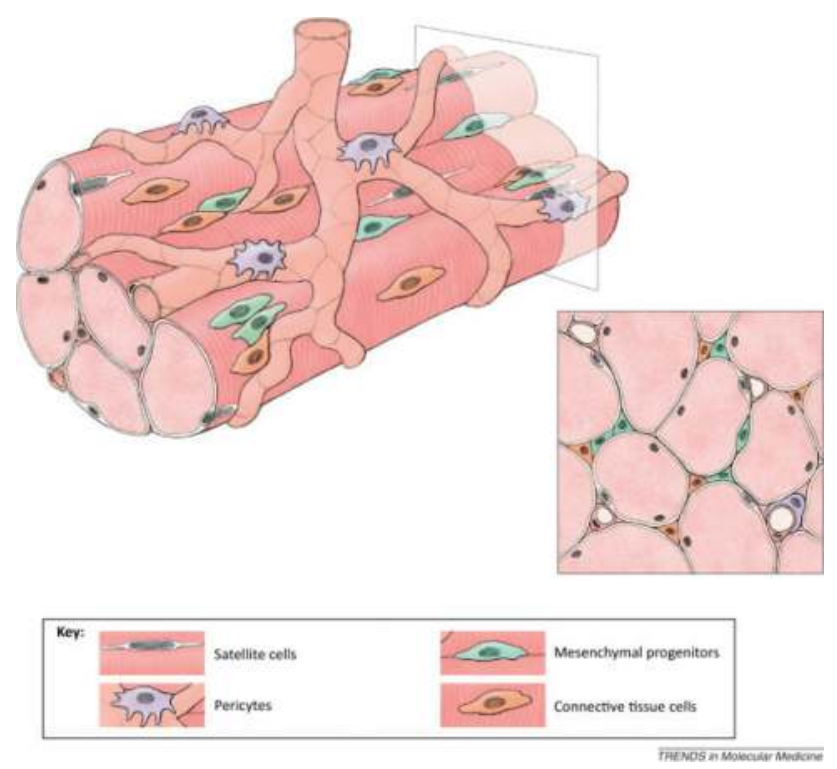

Figure 1. Satellite cells (white) constitute the major muscle stem cell population that reside beneath the basal lamina near the vasculature. Other muscle-resident stem cell populations such as pericytes (purple) and mesenchymal progenitors (green) contribute to muscle repair and regeneration. Reproduced with permission from Elsevier Ltd. Pannerec A, Marazzi G, Sassoon D. Stem cells in the hood: the skeletal muscle niche. Trends in Molecular Medicin; 2012;18(10):599606. DOI:10.1016/j.molmed.2012.07.004.

\subsection{Satellite cells}

Satellite cells $\left(\operatorname{Pax}^{+}\right)$reside in between the basal lamina and the sarcolemma in a quiescent state. They express integrin $\alpha 7 \beta 1$ receptors that bind with laminin in the basal lamina and M-cadherin adhesion molecules that interact with the sarcolemma [9]. In response to injury, they undergo asymmetrical cell-division and give rise to transit-amplifying cells and myoblasts that can either fuse with each other or with existing myofibers to initiate repair. The indispensable role of satellite cells in skeletal muscle regeneration has been well-documented using selective depletion of the $\mathrm{Pax}^{+}$cell population [17-20]. Interestingly, these studies also highlighted a role of Pax $7^{+}$satellite cells in the inhibition of fibrosis development by negative regulation of muscle-resident fibroblasts.

Besides Pax7, several markers have been used for the isolation of satellite cells such as VCAM-1 [21], $\alpha 7$ integrin [22], M-cadherin [23], and nestin [24]. However, it is unclear if these cell surface markers allow for the isolation of the same stem cell population. While the transplantation of autologous satellite cells seems like an effective therapeutic strategy, several challenges have been identified with this approach. For instance, the regenerative capacity of cultured satellite cells is significantly lower than freshly isolated cells [25]. Poor survival and migration of transplanted cells, as well as undesirable immune reactions, have also been 
reported [26, 27]. To circumvent these issues, alternative modes of satellite cell delivery have been attempted in preclinical studies. Collins et al. transplanted single intact myofibers into radiation-ablated muscles and demonstrated that as few as seven satellite cells associated with a single myofiber could generate $>100$ new myofibers containing thousands of myonuclei [28]. Other groups have shown beneficial effects of single fiber transplantation in preclinical models of aging [29] and heart failure [30]. However, the isolation of single myofibers is challenging and requires a high degree of technical skill and expertise as well as the need for regulatory approval. An alternative approach is to deliver bundles of minced muscle fibers to transfer not just satellite cells but also other cell types with an intact ECM. Corona et al. have repeatedly demonstrated appreciable muscle regeneration by transplantation of minced muscle autografts in both rodent [31-33] and porcine [34] models of volumetric muscle loss (VML).

A scaffold-based strategy for satellite cell encapsulation and delivery has also been attempted in various studies. Rossi et al. reported improvement in muscle structure, the total number of new myofibers as well as muscle function by delivering satellite cells encapsulated in a hyaluronan-based photocrosslinkable hydrogel to partially ablated tibialis anterior muscles [35]. Recent work by Corona et al. has evaluated the myoconductive properties of collagen gels [36], and laminin-111 supplemented hyaluronic acid hydrogels [37] by co-delivering them with minced muscle autografts in rodent models of VML. However, none of these strategies were able to augment the regenerative potential of minced muscle autografts, possibly due to an exacerbated immune response to the implanted materials or due to the inability of the materials to influence satellite cell activity.

\subsection{Mesenchymal stem cells}

MSCs are a multipotent stem cell population located in several adult tissues. From a tissue engineering standpoint, MSCs exhibit several attractive features such as self-renewal, multipotency, immunomodulation, and the secretion of a wide variety of pro-regenerative growth factors and cytokines. While MSCs can undergo differentiation into osteogenic, chondrogenic, adipogenic, and myogenic lineages in vitro under specific culture conditions, their capacity for site-specific differentiation post-transplantation into injured tissues is controversial [16]. Regardless, 646 on-going clinical trials are currently investigating the therapeutic efficacy of MSCs for a wide-range of different conditions in various tissues (source: clinicaltrials.gov).

Muscle-resident MSCs, isolated as $\mathrm{Sca}^{+} \mathrm{CD}^{-} 5^{-}$cells, are reported to be non-myogenic, as they do not form myotubes in culture or fuse with myofibers in vivo. However, intramuscular delivery of this cell population can increase Pax $7^{+}$satellite cell quantity, new fiber synthesis, myofiber hypertrophy, and arteriogenesis in eccentrically exercised mouse hindlimb muscles [38-40]. Muscle-derived MSCs are also known to secrete a wide variety of growth factors in response to exercise in vivo [38] and mechanical strain in vitro [41, 42]. Overall, these studies indicate that muscle-resident MSCs serve as a crucial component of the cellular niche that can facilitate muscle regeneration.

In clinical and preclinical studies, bone marrow remains the most commonly utilized source for the isolation of MSCs. Intramuscular delivery of bone marrow-derived MSCs (BMMC) into rodent models of crush trauma has improved muscle function in a dose-dependent 
manner $[43,44]$. Additionally, systemic delivery of BMMC via intra-arterial transplantation was also reported to restore muscle functional capacity in a rodent model of crush trauma. Since systemically delivered cells were not found in the traumatized muscle tissue, the authors speculated that the observed improvements in muscle function were likely due to the secretion of soluble factors by the transplanted cells [45]. Pumberger et al. [46] delivered BMMC with recombinant growth factors in an alginate cryogel in traumatized soleus muscles. This biomaterial driven approach enhanced paracrine signaling in MSCs, which resulted in improved muscle function, remodeled scar tissue, and increased the formation of new myofibers.

Corona et al. worked with a lineage depleted ( Lin $\left.^{-}\right)$fraction of bone marrow cells to increase the concentration of stem and progenitor cells in the isolated cell population. In a mouse model of ischemia-reperfusion (I/R) injury, the $\mathrm{Lin}^{-}$bone marrow-derived stem cells were injected intramuscularly. While the cells survived transplantation for up to 1 month after injury, they did not improve muscle function [47]. In a subsequent study, these cells were delivered intravenously in a mouse model of $\mathrm{I} / \mathrm{R}$ injury to avoid potential muscle damage from intramuscular injections. This approach resulted in stem cell homing to the injured leg and improvements in muscle regeneration as well as function [48]. However, the exact mechanism of repair or the contribution of specific stem cell subsets remains unclear.

In a muscle model of repeated laceration injury, BMMC were suspended in Matrigel and transplanted in the soleus muscles [49]. The BMMC treated muscles showed fewer fibers with a centrally located nucleus, and larger muscle fiber cross-sectional area compared to nontreated muscles, but no differences in fibrotic tissue deposition were observed. The study did not determine whether the BMMC participated in muscle regeneration directly by differentiating into myogenic cells, or indirectly through the secretion of angiogenic and proregenerative soluble mediators. In another study, it was demonstrated that transplantation of BMMC suspended in a fibrin matrix in a rodent model of muscle laceration restored muscle function. Again, the cells did not differentiate into or fuse with skeletal myofibers, signifying an alternate mechanism for repair [50]. Currently, the trophic support provided by MSCs (via the release of soluble growth factors and cytokines) is increasingly being considered as the primary mechanism for tissue repair and regeneration. This prevailing hypothesis has provided the underlining foundation for a plethora of clinical trials.

\subsection{Adipose-derived stem cells}

Adipose-derived stem cells (ASCs) are morphologically and phenotypically similar to MSCs and have gained increased popularity in regenerative medicine because large quantities can be easily isolated using minimally invasive procedures [51]. ASCs are reported to exhibit myogenic potential in vitro [52]. Myogenic progenitors derived from ASC cultures have successfully engrafted into skeletal muscle, promoted myofiber synthesis, and restored dystrophin expression in dystrophic $(m d x)$ mice [53]. Besides improving muscle regeneration, ASCs can also modulate inflammation and fibrosis to ameliorate the dystrophic phenotype in mouse models [54]. To combat fibrosis development in injured dystrophic muscles, ASCs were delivered in combination with an anti-fibrotic medication called losartan. The combined treatment was able to downregulate TGF- $\beta 1$, inhibit fibrosis, and simultaneously 
improved muscle regeneration and hypertrophy [55]. In another study, transplantation of ASCs alleviated skeletal muscle fibrosis induced by high dose radiation by suppressing the level of TGF- $\beta 1$ [56]. In a VML model, ASCs were delivered to the defect site in a collagen hydrogel. The results showed that this treatment approach accelerated muscle repair and vascularization while simultaneously reducing inflammation and fibrosis [57]. Collectively, these studies suggest that ASCs can support muscle repair and regeneration either directly by giving rise to myogenic progenitors or indirectly by attenuating inflammation and fibrosis.

\subsection{Hematopoietic stem cells}

HSCs are multipotent stem cells that continuously replenish all classes of blood cells including both the lymphoid and the myeloid lineages. Cells of the adaptive immune system comprise the lymphoid lineage while cells of the innate immune system, megakaryocytes, and erythrocytes comprise the myeloid lineage [58]. Adult bone marrow, cord blood, and mobilized peripheral blood are the primary sources of HSCs used in clinical transplantation protocols for the treatment of cancer and other blood or immune disorders [59].

The first report of bone marrow-derived cell-mediated muscle regeneration in vivo was published in 1998 by Ferrari et al. [60]. Since then several studies have repeatedly shown that bone marrow-derived cells can contribute to myogenesis and muscle regeneration. For instance, Gussoni et al. [61] showed that HSCs derived from wild-type mice could participate in myogenesis and partially restore dystrophin expression in lethally irradiated $m d x$ mice following intravenous transplantation. LaBarge et al. [62] demonstrated that following irradiationinduced depletion of satellite cells, cells from a bone marrow transplant could occupy the satellite cell niche and contribute to myofiber regeneration. In a recent report, Goldman et al. [63] transplanted bone-marrow derived mononuclear cells in a rat model of VML. The transplanted cells contributed to limited de novo muscle fiber regeneration, without any significant changes in myogenic gene transcription and muscle function.

Other studies have highlighted the role of injury in HSC-mediated myogenic repair. It has repeatedly been shown that HSCs contribute to myogenic events only in response to damage or injury, but do not participate in myofiber repair under normal physiological conditions. For instance, Corbel et al. [64] showed that although the fusion of hematopoietic progenitors with myofibers could occur at low frequency under normal physiological conditions, this capacity increases significantly with muscle damage. Polesskaya et al. [65] observed a 10-fold increase in the numbers of cells coexpressing the cell surface markers CD45 and Sca1 following cardiotoxin injury in skeletal muscle. It was further demonstrated that while the $\mathrm{CD} 45^{+}$cell population from uninjured muscle did not differentiate into myogenic cells, the $\mathrm{CD} 45^{+}$cells from injured muscle readily underwent myogenic differentiation in vitro. The myogenic commitment of this cell population was induced by the stimulation of the Wnt signaling cascade.

In contrast, Camargo et al. [66] suggested that in response to muscle injury, myeloid inflammatory progenitors derived from HSCs are recruited to the site of muscle damage and undergo stochastic fusion with regenerating myofibers. Due to these conflicting reports, it 
remains unclear if the HSCs can give rise to myogenic cells in response to environmental cues or if they undergo nuclear reprogramming post-fusion with a damaged myofiber.

The hematopoietic capacity of adult muscle-resident stem cells has also been investigated. Jackson et al. [67] isolated muscle-resident stem cells by enzymatic digestion and co-transplanted them into irradiated mice with whole bone marrow cells. After 6-12 weeks the recipient mice showed engraftment of muscle-derived cells in the bone-marrow and reconstitution of all the major blood cell lineages. Therefore, endogenous adult stem cells resident in skeletal muscle may represent a multipotent stem cell population capable of giving rise to both blood and muscle tissue.

Taken together, these studies indicate that adult stem cells may have differentiation potential beyond their tissue of origin. It further calls into question the influence of environment versus lineage in the commitment and differentiation of stem cell populations and highlights a previously unrecognized potential for plasticity in tissue-resident adult stem cells.

\subsection{Pericytes}

Pericytes are perivascular stem cells that encase and form intimate connections with adjacent capillary endothelial cells [68]. They influence the migration, proliferation, permeability, and contractility of endothelial cells and play essential roles in various stages of angiogenesis [69-71]. These cells can also regulate vascular diameter and capillary blood flow by producing both vasoconstriction and vasodilation within capillary beds [72]. Two major subpopulations of pericytes have been identified: type $1\left(\mathrm{Nestin}^{-} \mathrm{NG}^{+}\right)$and type $2\left(\mathrm{Nestin}^{+} \mathrm{NG} 2^{+}\right)$. While type 1 pericytes are reported to contribute to fat accumulation, type 2 pericytes are known to support new muscle formation [73].

Recent reports have suggested an indispensable role of pericytes in the postnatal growth of skeletal muscle [74, 75]. Type 2 pericytes have been identified in the satellite cell niche and are believed to be the primary population involved in muscle formation [73]. Kostallari et al. [76] showed that pericyte depletion could result in significant myofiber hypotrophy with a slight increase in a total number of Pax $7^{+}$satellite cells. In vitro co-culture studies demonstrated that pericytes could promote both myogenic differentiation and quiescence in satellite cells through the secretion of insulin-like growth factor 1 (IGF-1) and angiopoietin 1 (ANGPT1), respectively [76]. Dellavalle et al. [75] showed that vascular pericytes are bi-potent, as they give rise to both the smooth muscle layer of blood vessels and skeletal muscle fibers, during postnatal growth in mice. They also demonstrated that pericytes express myogenic markers only in differentiated myotubes, and when transplanted into immunodeficient dystrophic (scid-mdx) mice, pericytes can generate dystrophin-positive myofibers [77]. However, the environmental or biochemical signals that regulate the myogenic differentiation of pericytes remain unknown. This study also demonstrated that pericytes delivered systemically can cross the vessel wall to colonize skeletal muscle - a feature that is absent in satellite cells. Lorant et al. [78] were able to corroborate these findings by injecting human perivascular stem cells into cryoinjured muscles of scid mice. The cells were able to integrate into the host injured tissue and were associated with the production of structural proteins expressed in differentiated myofibers. 
Meng et al. also attempted to replicate these studies by delivering muscle-derived pericytes intraarterially in scid-mdx mice [79]. Surprisingly, their results showed that pericytes did not contribute to muscle regeneration. They attributed the discrepancies between their findings and previous data to the differences in cell-isolation and culture protocols, animal models, and outcome measurements. Therefore, these studies suggest that while pericytes may represent a useful cell population for future cell therapy of musculoskeletal disorders, more comprehensive studies are needed to establish a clear and definitive role for these cells in muscle regeneration and repair.

\section{Extracellular matrix for skeletal muscle regeneration}

The ECM in skeletal muscle is critical for tissue development, structural support, and force transmission [10]. The main components of ECM are largely conserved across animal species $[80,81]$. The ECM of skeletal muscle is organized into three layers; the endomysium layer surrounds the individual muscle fibers, the perimysium surrounds the bundles of muscle fibers known as fascicles, and the epimysium surrounds the entire muscle (Figure 2). Collagen type I is predominant in the perimysium, whereas collagen type III is prevalent in the endomysium

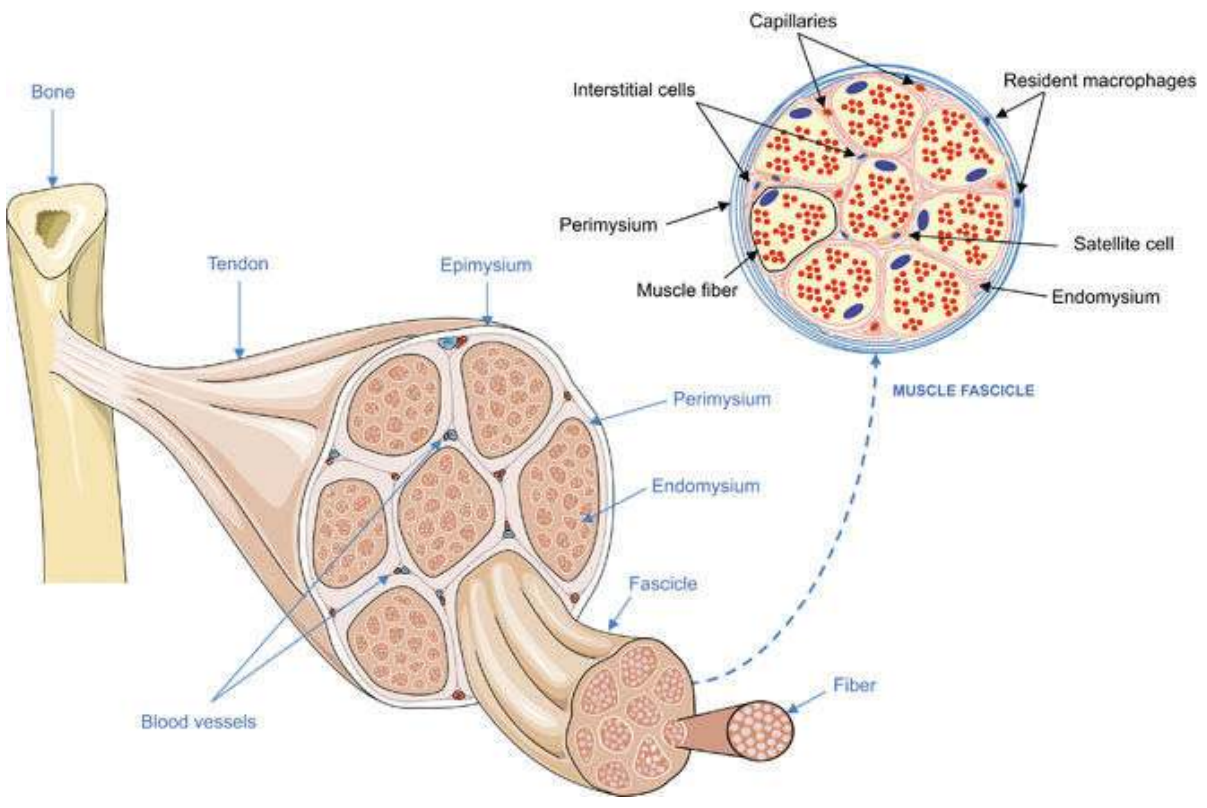

Figure 2. Skeletal muscle consists of muscle fibers bound by connective tissue. The outermost sheath of connective tissue that wraps around the muscle is called the epimysium. Bundles of muscle fibers, called fascicles, are enclosed by the perimysium and each muscle fiber is covered in a thin connective tissue layer called the endomysium that contains the extracellular fluid and nutrients to support the muscle fiber. Reproduced with permission from Springer Nature. Sciorati, C., et al., Cell death, clearance and immunity in the skeletal muscle. Cell Death and Differentiation. 2016;23(6):927-937. DOI: $10.1038 /$ cdd.2015.171. 
and the epimysium [82]. The basement membrane is a specialized layer of ECM between the sarcolemma of a muscle fiber and the surrounding endomysium [83]. Laminin and collagen type IV form the principal ECM components of the basal lamina layer [84]. The reticular lamina, located below the basement membrane, is composed mainly of fibrils of collagens (type I, III, and VI) and fibronectin in a proteoglycan-rich gel [83, 85].

Two major transmembrane protein complexes in the muscle fiber membrane are responsible for force transmission from the outer ECM to the inner cytoskeleton: the dystroglycan/sarcoglycan complex and the integrin $\alpha 7$-laminin complex [86]. These linkages are crucial for the stabilization of sarcolemma during contraction and are important for normal muscle function and strength. The importance of these linkages is demonstrated by the fact that defects in these molecules can result in the development of muscular dystrophy [87, 88]. A list of essential extracellular and intracellular components identified in skeletal muscle is provided in Table 1.

Stem cells in skeletal muscle are sensitive to biochemical and biophysical cues provided by the ECM [89]. For instance, loss of regenerative capacity in laminin-deficient $(d y / d y)$ mice, as well as enhanced satellite cell activity with laminin-111 supplementation in vivo, suggest a pivotal role for this ECM protein in the regulation of stem cell function post-injury [90-92]. Similarly, the absence of collagen VI in Col6a ${ }^{-1-}$ mice can impair satellite cell self-renewal and repair following injury $[93,94]$. Conversely, stem cells can also influence ECM composition. While fibroblasts are considered the leading contributor of ECM production in the skeletal

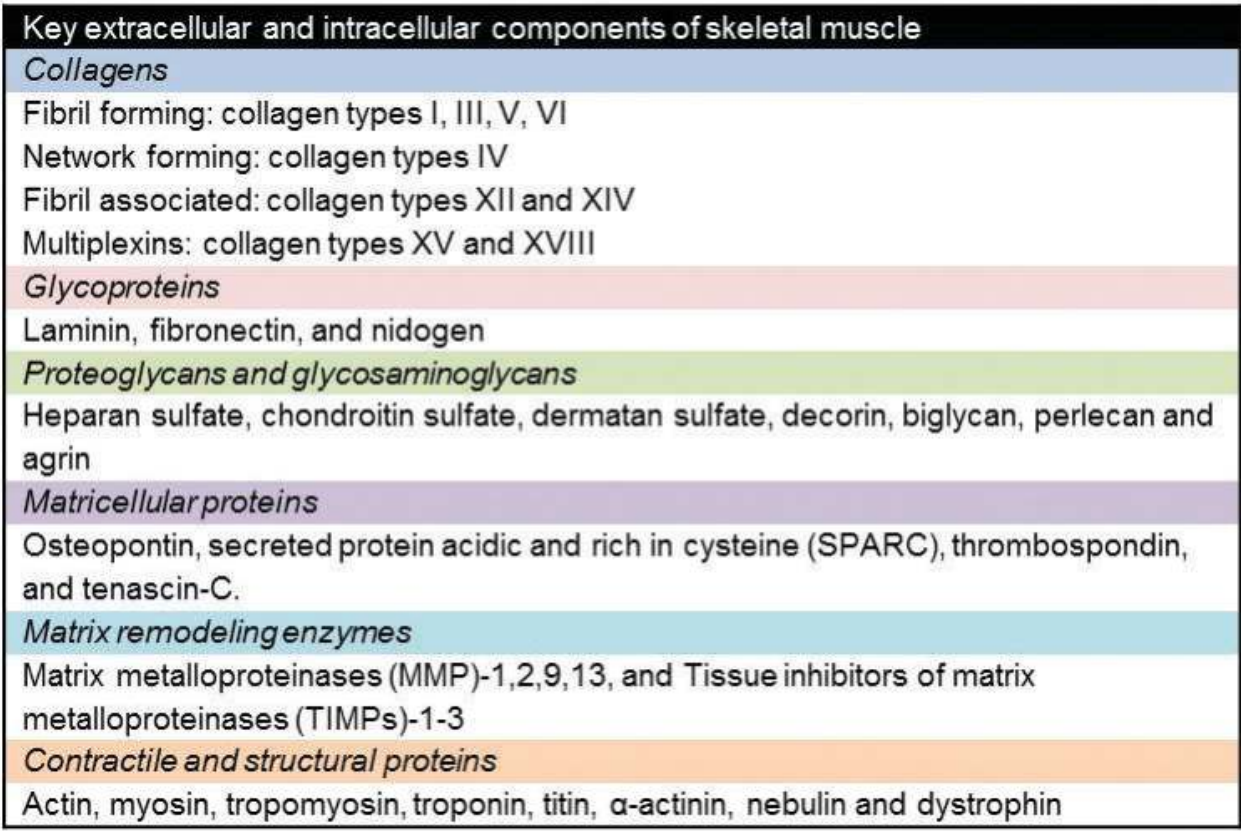

Table 1. The major extracellular and intracellular components responsible for regulating skeletal muscle function [126]. 
muscle, stem cells including satellite cells and MSCs can also produce several ECM constituents including collagen, laminin, fibronectin, and matrix metalloproteinases [12, 42, 95-97]. Thus, ECM not only serves as a supportive framework for skeletal muscle, but also dynamically regulates resident cell activity in a way that can direct tissue development, repair, remodeling, and function [89].

\subsection{ECM scaffolds for skeletal muscle repair}

Challenges associated with cell-centric therapies such as low survival, poor engraftment, inadequate supply of donor cells, and culture-induced changes in differentiation potential have motivated the development of acellular ECM-based tissue engineered therapeutic strategies. Biologic ECM scaffolds are prepared by the removal of cellular antigens and are at the forefront of tissue engineering strategies for muscle repair. These decellularized scaffolds preserve the ultrastructure and composition of the ECM [81] and are known to contain basement membrane structural proteins, growth factors, and glycosaminoglycans (GAG). Therefore, these scaffolds possess the potential to recruit endogenous host cells while evading the problems associated with the delivery of exogenous cells such as cellular apoptosis, immunogenicity and ineffective delivery $[98,99]$.

In preclinical models, these scaffolds are reported to support vascularization and functional recovery post-VML injury [100-105]. While some studies have used skeletal muscle [102, 106] as the source of ECM, other studies have created decellularized scaffolds from dermis [104, 107], porcine urinary bladder (UBM) [108-110], and small intestinal mucosa (SIS) [104, 111, 112] for skeletal muscle tissue engineering. Additionally, scaffolds composed of single ECM proteins such as collagen [113], laminin-111 [37, 114], and cell-derived ECM such as Matrigel ${ }^{\mathrm{TM}}[115,116]$ have also been used for skeletal muscle repair and regeneration.

Degradation products of ECM scaffolds are known to exert chemotactic and mitogenic effects on multipotential progenitor cells in vitro [117] and in vivo [118]. The recruitment of several stem cell populations such as Sca1 ${ }^{+}$cells $[32,119]$, perivascular stem cells [120, 121], pluripotent adult progenitors $\left(\right.$ Sox $\left.^{+}\right)$[122], CD133+ progenitor cells [123], and neural stem cells [124] has been reported post-implantation of ECM scaffolds in animal models. However, the ability of these scaffolds to recruit sufficient quantities of satellite cells to promote their activity in the VML defect region has not been conclusively established [32, 109]. For instance, Corona et al. reported that co-delivery of decellularized urinary bladder matrix negatively affects the regenerative capacity of minced muscle autografts in a rodent model of VML [108]. Using a similar approach, Kasukonis et al. implanted an allogeneic decellularized skeletal muscle scaffold with minced muscle autografts in a rat model of VML [125]. In contrast to the findings reported by Corona et al., they demonstrated significant improvements in muscle mass and peak contractile force.

\subsection{Clinical application of ECM scaffolds}

Recent work has also described encouraging clinical outcomes following the implantation of decellularized scaffolds in patients with VML injuries [126]. In a five patient cohort study, the VML injured muscle compartment was reconstructed with a commercially available ECM 
bioscaffold (MatriStem, Acell Inc.) [98]. Within 24-48 hours after surgery, each patient was placed in an aggressive and exhaustive physical therapy program, as the application of a mechanical load during the ECM remodeling phase is reported to promote cellular infiltration, angiogenesis, and connective tissue reorganization and alignment. While this strategy promoted perivascular stem cell infiltration and angiogenesis at the site of scaffold implantation, significant muscle regeneration was not observed. Histological images indicated the presence of small islands of desmin ${ }^{+}$and myosin heavy chain $\left(\mathrm{MHC}^{+}\right)$myofibers separated from the adjacent healthy muscle by collagenous connective tissue. Six months after surgery, three of five subjects showed improvement in the functional outcomes [98].

In a subsequent report [127], six patients were implanted with MatriStem (UBM-ECM, Acell Inc.), and an additional seven patients were implanted with BioDesign (SIS-ECM, Cook Medical) or XenMatrix (dermis-ECM, C.R. Bard). Similar to the previous study, post-surgical physical therapy was initiated between 24 and 48 hours following surgery. In addition to perivascular stem cell infiltration and desmin ${ }^{+}$islands of regenerated myofibers, this study also reported the presence of neurogenic cells ( $\beta$-III tubulin ${ }^{+}$) at the remodeling site. Ultrasound imaging revealed complete resorption of the BioDesign and MatriStem scaffold materials by seven months. Out of 13 patients, 7 had improvement from pre-surgical maximum strength with an average force production change of $\sim 15.2 \%$ at $6-8$ weeks post-surgery. By 24-28 weeks, an average improvement of $\sim 37.3 \%$ was reported.

In the aforementioned studies, due to the lack of significant muscle regeneration, the improvements in muscle function could be attributed to both "functional fibrosis" [102] and physical rehabilitation [128]. Although the exact mechanism by which ECM scaffolds support tissue remodeling is unclear, it has been associated with recruitment of stem/progenitor cells. While these studies do not provide conclusive evidence of a causal relationship between recruitment of stem cells and improved functional outcomes, the reported findings suggest that ECMbased scaffolds are worthy of investigation as a viable treatment option for traumatic muscle injuries. Future studies could augment the regenerative potential of these scaffolds by simultaneous delivery of autologous stem cell populations or a more targeted rehabilitation program.

\section{Conclusion}

Muscle regeneration and recovery is a complex process that involves several different stem cell populations and ECM components. While the delivery of stem cells in injured or dystrophic muscles has been associated with improvements in muscle repair and function, the exact mechanism by which these cells contribute to muscle repair is unclear. Future studies should focus on identification of the intrinsic and extrinsic regulatory mechanisms that govern satellite and non-satellite cell differentiation and trophic factor secretion during the muscle regeneration process. Additionally, the mechanical and biochemical cues provided by key ECM components that can promote or dysregulate stem cell activity should be examined. This information will be crucial in the discovery of biomaterial-based strategies to augment the stem cell mediated muscle repair. 


\section{Author details}

Andrew Dunn, Madison Marcinczyk, Muhamed Talovic, Krishna Patel, Gabriel Haas and Koyal Garg*

*Address all correspondence to: koyal.garg@slu.edu

Department of Biomedical Engineering, Parks College of Engineering, Aviation, and Technology, Saint Louis University, St. Louis, MO, USA

\section{References}

[1] Mann CJ et al. Aberrant repair and fibrosis development in skeletal muscle. Skeletal Muscle. 2011;1(1):21

[2] Baoge L et al. Treatment of skeletal muscle injury: A review. ISRN Orthopedics. 2012;2012:689012

[3] Garg K et al. Therapeutic strategies for preventing skeletal muscle fibrosis after injury. Frontiers in Pharmacology. 2015;6:87

[4] Garg K et al. Volumetric muscle loss: Persistent functional deficits beyond frank loss of tissue. Journal of Orthopaedic Research. 2015;33(1):40-46

[5] Hurtgen BJ et al. Severe muscle trauma triggers heightened and prolonged local musculoskeletal inflammation and impairs adjacent tibia fracture healing. Journal of Musculoskeletal \& Neuronal Interactions. 2016;16(2):122-134

[6] Lieber RL et al. Cellular mechanisms of tissue fibrosis. 4. Structural and functional consequences of skeletal muscle fibrosis. American Journal of Physiology. Cell Physiology. 2013;305(3):C241-C252

[7] Smith C et al. The inflammatory response to skeletal muscle injury: Illuminating complexities. Sports Medicine. 2008;38(11):947-969

[8] Emery AE. The muscular dystrophies. Lancet. 2002;359(9307):687-695

[9] Kuang $S$ et al. Niche regulation of muscle satellite cell self-renewal and differentiation. Cell Stem Cell. 2008;2(1):22-31

[10] Thomas $\mathrm{K}$ et al. Extracellular matrix regulation in the muscle satellite cell niche. Connective Tissue Research. 2015;56(1):1-8

[11] Challen GA et al. A side order of stem cells: The SP phenotype. Stem Cells. 2006;24(1):3-12

[12] Motohashi N et al. Muscle CD31(-) CD45(-) side population cells promote muscle regeneration by stimulating proliferation and migration of myoblasts. The American Journal of Pathology. 2008;173(3):781-791 
[13] Uezumi A et al. Mesenchymal progenitors distinct from satellite cells contribute to ectopic fat cell formation in skeletal muscle. Nature Cell Biology. 2010;12(2):143-152

[14] Joe AW et al. Muscle injury activates resident fibro/adipogenic progenitors that facilitate myogenesis. Nature Cell Biology. 2010;12(2):153-163

[15] Cottle BJ et al. Skeletal muscle-derived interstitial progenitor cells (PICs) display stem cell properties, being clonogenic, self-renewing, and multi-potent in vitro and in vivo. Stem Cell Research \& Therapy. 2017;8(1):158

[16] Boppart MD et al. Defining a role for non-satellite stem cells in the regulation of muscle repair following exercise. Frontiers in Physiology. 2013;4:310

[17] Sambasivan R et al. Pax7-expressing satellite cells are indispensable for adult skeletal muscle regeneration. Development. 2011;138(17):3647-3656

[18] McCarthy JJ et al. Effective fiber hypertrophy in satellite cell-depleted skeletal muscle. Development. 2011;138(17):3657-3666

[19] Relaix F et al. Satellite cells are essential for skeletal muscle regeneration: The cell on the edge returns centre stage. Development. 2012;139(16):2845-2856

[20] Murphy MM et al. Satellite cells, connective tissue fibroblasts and their interactions are crucial for muscle regeneration. Development. 2011;138(17):3625-3637

[21] Rosen GD et al. Roles for the integrin VLA-4 and its counter receptor VCAM-1 in myogenesis. Cell. 1992;69(7):1107-1119

[22] Blanco-Bose WE et al. Purification of mouse primary myoblasts based on alpha 7 integrin expression. Experimental Cell Research. 2001;265(2):212-220

[23] Irintchev A et al. Expression pattern of M-cadherin in normal, denervated, and regenerating mouse muscles. Developmental Dynamics. 1994;199(4):326-337

[24] Day K et al. Nestin-GFP reporter expression defines the quiescent state of skeletal muscle satellite cells. Developmental Biology. 2007;304(1):246-259

[25] Montarras D et al. Direct isolation of satellite cells for skeletal muscle regeneration. Science. 2005;309(5743):2064-2067

[26] Cossu G et al. New therapies for muscular dystrophy: Cautious optimism. Trends in Molecular Medicine. 2004;10(10):516-520

[27] Tedesco FS et al. Repairing skeletal muscle: Regenerative potential of skeletal muscle stem cells. The Journal of Clinical Investigation. 2010;120(1):11-19

[28] Collins CA et al. Stem cell function, self-renewal, and behavioral heterogeneity of cells from the adult muscle satellite cell niche. Cell. 2005;122(2):289-301

[29] Hall JK et al. Prevention of muscle aging by myofiber-associated satellite cell transplantation. Science Translational Medicine. 2010;2(57):57ra83 
[30] Suzuki K et al. Single fibers of skeletal muscle as a novel graft for cell transplantation to the heart. The Journal of Thoracic and Cardiovascular Surgery. 2002;123(5):984-992

[31] Corona BT et al. Autologous minced muscle grafts: A tissue engineering therapy for the volumetric loss of skeletal muscle. American Journal of Physiology. Cell Physiology. 2013;305(7):C761-C775

[32] Garg K et al. Transplantation of devitalized muscle scaffolds is insufficient for appreciable de novo muscle fiber regeneration after volumetric muscle loss injury. Cell and Tissue Research. 2014;358(3):857-873

[33] Hurtgen BJ et al. Autologous minced muscle grafts improve endogenous fracture healing and muscle strength after musculoskeletal trauma. Physiological Reports. 2017;5(14): e13362

[34] Ward CL et al. Autologous minced muscle grafts improve muscle strength in a porcine model of volumetric muscle loss injury. Journal of Orthopaedic Trauma. 2016;30(12): e396-e403

[35] Rossi CA et al. In vivo tissue engineering of functional skeletal muscle by freshly isolated satellite cells embedded in a photopolymerizable hydrogel. The FASEB Journal. 2011;25(7):2296-2304

[36] Ward CL et al. An autologous muscle tissue expansion approach for the treatment of volumetric muscle loss. Bioresearch Open Access. 2015;4(1):198-208

[37] Goldman SM et al. Co-delivery of a laminin-111 supplemented hyaluronic acid based hydrogel with minced muscle graft in the treatment of volumetric muscle loss injury. PLoS One. 2018;13(1):e0191245

[38] Huntsman HD et al. Mesenchymal stem cells contribute to vascular growth in skeletal muscle in response to eccentric exercise. American Journal of Physiology. Heart and Circulatory Physiology. 2013;304(1):H72-H81

[39] Valero MC et al. Eccentric exercise facilitates mesenchymal stem cell appearance in skeletal muscle. PLoS One. 2012;7(1):e29760

[40] Zou K et al. Mesenchymal stem cells augment the adaptive response to eccentric exercise. Medicine and Science in Sports and Exercise. 2015;47(2):315-325

[41] Huntsman HD et al. The impact of mechanically stimulated muscle-derived stromal cells on aged skeletal muscle. Experimental Gerontology. 2017;103:35-46

[42] De Lisio $\mathrm{M}$ et al. Substrate and strain alter the muscle-derived mesenchymal stem cell secretome to promote myogenesis. Stem Cell Research \& Therapy. 2014;5(3):74

[43] Winkler $\mathrm{T}$ et al. Dose-response relationship of mesenchymal stem cell transplantation and functional regeneration after severe skeletal muscle injury in rats. Tissue Engineering. Part A. 2009;15(3):487-492

[44] Matziolis G et al. Autologous bone marrow-derived cells enhance muscle strength following skeletal muscle crush injury in rats. Tissue Engineering. 2006;12(2):361-367 
[45] von Roth P et al. Intra-arterial MSC transplantation restores functional capacity after skeletal muscle trauma. The Open Orthopaedics Journal. 2012;6:352-356

[46] Pumberger M et al. Synthetic niche to modulate regenerative potential of MSCs and enhance skeletal muscle regeneration. Biomaterials. 2016;99:95-108

[47] Corona BT et al. Intramuscular transplantation and survival of freshly isolated bone marrow cells following skeletal muscle ischemia-reperfusion injury. Journal of Trauma and Acute Care Surgery. 2013;75(2 Suppl 2):S142-S149

[48] Corona BT et al. Accelerated functional recovery after skeletal muscle ischemia-reperfusion injury using freshly isolated bone marrow cells. The Journal of Surgical Research. 2014;188(1):100-109

[49] Andrade BM et al. Bone marrow mesenchymal cells improve muscle function in a skeletal muscle re-injury model. PLoS One. 2015;10(6):e0127561

[50] Natsu K et al. Allogeneic bone marrow-derived mesenchymal stromal cells promote the regeneration of injured skeletal muscle without differentiation into myofibers. Tissue Engineering. 2004;10(7-8):1093-1112

[51] Gimble JM et al. Adipose-derived stem cells for regenerative medicine. Circulation Research. 2007;100(9):1249-1260

[52] Mizuno $\mathrm{H}$ et al. Myogenic differentiation by human processed lipoaspirate cells. Plastic and Reconstructive Surgery. 2002;109(1):199-209; discussion 210-1

[53] Zhang $Y$ et al. Long-term engraftment of myogenic progenitors from adipose-derived stem cells and muscle regeneration in dystrophic mice. Human Molecular Genetics. 2015;24(21):6029-6040

[54] Pinheiro $\mathrm{CH}$ et al. Local injections of adipose-derived mesenchymal stem cells modulate inflammation and increase angiogenesis ameliorating the dystrophic phenotype in dystrophin-deficient skeletal muscle. Stem Cell Reviews. 2012;8(2):363-374

[55] Lee EM et al. Therapeutic effects of mouse adipose-derived stem cells and losartan in the skeletal muscle of injured mdx mice. Cell Transplantation. 2015;24(5):939-953

[56] Sun W et al. Adipose-derived stem cells alleviate radiation-induced muscular fibrosis by suppressing the expression of TGF-beta1. Stem Cells International. 2016;2016:5638204

[57] Huang $\mathrm{H}$ et al. Preferred M2 polarization by ASC-based hydrogel accelerated angiogenesis and myogenesis in volumetric muscle loss rats. Stem Cells International. 2017;2017:2896874

[58] Kondo M. Lymphoid and myeloid lineage commitment in multipotent hematopoietic progenitors. Immunological Reviews. 2010;238(1):37-46

[59] Huang S et al. Candidate hematopoietic stem cells from fetal tissues, umbilical cord blood vs. adult bone marrow and mobilized peripheral blood. Experimental Hematology. 1998;26(12):1162-1171 
[60] Ferrari G et al. Muscle regeneration by bone marrow-derived myogenic progenitors. Science. 1998;279(5356):1528-1530

[61] Gussoni E et al. Dystrophin expression in the mdx mouse restored by stem cell transplantation. Nature. 1999;401(6751):390-394

[62] LaBarge MA et al. Biological progression from adult bone marrow to mononucleate muscle stem cell to multinucleate muscle fiber in response to injury. Cell. 2002;111(4):589-601

[63] Goldman SM et al. Evaluation of bone marrow mononuclear cells as an adjunct therapy to minced muscle graft for the treatment of volumetric muscle loss injuries. Stem Cell Research \& Therapy. 2017;8(1):142

[64] Corbel SY et al. Contribution of hematopoietic stem cells to skeletal muscle. Nature Medicine. 2003;9(12):1528-1532

[65] Polesskaya A et al. Wnt signaling induces the myogenic specification of resident CD45+ adult stem cells during muscle regeneration. Cell. 2003;113(7):841-852

[66] Camargo FD et al. Single hematopoietic stem cells generate skeletal muscle through myeloid intermediates. Nature Medicine. 2003;9(12):1520-1527

[67] Jackson KA et al. Hematopoietic potential of stem cells isolated from murine skeletal muscle. Proceedings of the National Academy of Sciences of the United States of America. 1999;96(25):14482-14486

[68] Birbrair A et al. Pericytes: Multitasking cells in the regeneration of injured, diseased, and aged skeletal muscle. Frontiers in Aging Neuroscience. 2014;6:245

[69] Armulik A et al. Endothelial/pericyte interactions. Circulation Research. 2005;97(6): 512-523

[70] Dulmovits BM et al. Microvascular remodeling and wound healing: A role for pericytes. The International Journal of Biochemistry \& Cell Biology. 2012;44(11):1800-1812

[71] Ribatti D et al. The role of pericytes in angiogenesis. The International Journal of Developmental Biology. 2011;55(3):261-268

[72] Rucker HK et al. Cellular mechanisms of CNS pericytes. Brain Research Bulletin. 2000;51(5):363-369

[73] Birbrair A et al. Role of pericytes in skeletal muscle regeneration and fat accumulation. Stem Cells and Development. 2013;22(16):2298-2314

[74] Birbrair A et al. Pericytes are essential for skeletal muscle formation. Stem Cell Reviews. 2015;11(4):547-548

[75] Dellavalle A et al. Pericytes resident in postnatal skeletal muscle differentiate into muscle fibres and generate satellite cells. Nature Communications. 2011;2:499

[76] Kostallari E et al. Pericytes in the myovascular niche promote post-natal myofiber growth and satellite cell quiescence. Development. 2015;142(7):1242-1253 
[77] Dellavalle A et al. Pericytes of human skeletal muscle are myogenic precursors distinct from satellite cells. Nature Cell Biology. 2007;9(3):255-267

[78] Lorant J et al. Skeletal muscle regenerative potential of human Mu stem cells following transplantation into injured mice muscle. Molecular therapy; 2018;26(2):618-633

[79] Meng J et al. Contribution of human muscle-derived cells to skeletal muscle regeneration in dystrophic host mice. PLoS One. 2011;6(3):e17454

[80] Londono R et al. Biologic scaffolds for regenerative medicine: Mechanisms of in vivo remodeling. Annals of Biomedical Engineering. 2015;43(3):577-592

[81] Wolf MT et al. Naturally derived and synthetic scaffolds for skeletal muscle reconstruction. Advanced Drug Delivery Reviews. 2015;84:208-221

[82] Gillies AR et al. Structure and function of the skeletal muscle extracellular matrix. Muscle \& Nerve. 2011;44(3):318-331

[83] Garg Ketal. Influence of exercise and aging on extracellular matrix composition in the skeletal muscle stem cell niche. Journal of Applied Physiology (1985). 2016;121(5):1053-1058

[84] Kjaer M. Role of extracellular matrix in adaptation of tendon and skeletal muscle to mechanical loading. Physiological Reviews. 2004;84(2):649-698

[85] Sanes JR. The basement membrane/basal lamina of skeletal muscle. Journal of Biological Chemistry. 2003;278(15):12601-12604

[86] Grounds MD et al. Strength at the extracellular matrix-muscle interface. Scandinavian Journal of Medicine \& Science in Sports. 2005;15(6):381-391

[87] Mayer U et al. Absence of integrin alpha 7 causes a novel form of muscular dystrophy. Nature Genetics. 1997;17(3):318-323

[88] Bonilla E et al. Duchenne muscular dystrophy: Deficiency of dystrophin at the muscle cell surface. Cell. 1988;54(4):447-452

[89] Garg K et al. Influence of exercise and aging on extracellular matrix composition in the skeletal muscle stem cell niche. Journal of Applied Physiology; 2016;121(5):1053-1058

[90] Hodges BL et al. Altered expression of the alpha7beta1 integrin in human and murine muscular dystrophies. Journal of Cell Science. 1997;110(Pt 22):2873-2881

[91] Zou K et al. Laminin-111 improves skeletal muscle stem cell quantity and function following eccentric exercise. Stem Cells Translational Medicine. 2014;3(9):1013-1022

[92] Rooney JE et al. Laminin-111 protein therapy prevents muscle disease in the mdx mouse model for Duchenne muscular dystrophy. Proceedings of the National Academy of Sciences of the United States of America. 2009;106(19):7991-7996

[93] Bonnemann CG et al. Myopathies resulting from mutations in sarcomeric proteins. Current Opinion in Neurology. 2004;17(5):529-537 
[94] Urciuolo A et al. Collagen VI regulates satellite cell self-renewal and muscle regeneration. Nature Communications. 2013;4:1964

[95] Bentzinger CF et al. Fibronectin regulates Wnt7a signaling and satellite cell expansion. Cell Stem Cell. 2013;12(1):75-87

[96] Schuler F et al. Expression of laminin isoforms in mouse myogenic cells in vitro and in vivo. Journal of Cell Science. 1995;108(Pt 12):3795-3805

[97] Guerin CW et al. Synthesis and secretion of matrix-degrading metalloproteases by human skeletal muscle satellite cells. Developmental Dynamics. 1995;202(1):91-99

[98] Sicari BM et al. An acellular biologic scaffold promotes skeletal muscle formation in mice and humans with volumetric muscle loss. Science Translational Medicine. 2014;6(234):234ra58

[99] Elmashhady $\mathrm{H}$ et al. Decellularized extracellular matrices for tissue engineering applications. Electrospinnning. 2017;1(1):87-99

[100] Porzionato A et al. Decellularized human skeletal muscle as biologic scaffold for reconstructive surgery. International Journal of Molecular Sciences. 2015;16(7):14808-14831

[101] Wolf MT et al. Biologic scaffold composed of skeletal muscle extracellular matrix. Biomaterials. 2012;33(10):2916-2925

[102] Corona BT et al. The promotion of a functional fibrosis in skeletal muscle with volumetric muscle loss injury following the transplantation of muscle-ECM. Biomaterials. 2013;34(13):3324-3335

[103] Brown BN et al. Macrophage phenotype and remodeling outcomes in response to biologic scaffolds with and without a cellular component. Biomaterials. 2009;30(8):1482-1491

[104] Ma J et al. Investigating muscle regeneration with a dermis/small intestinal submucosa scaffold in a rat full-thickness abdominal wall defect model. Journal of Biomedical Materials Research. Part B, Applied Biomaterials. 2015;103(2):355-364

[105] Merritt EK et al. Functional assessment of skeletal muscle regeneration utilizing homologous extracellular matrix as scaffolding. Tissue Engineering. Part A. 2010;16(4): 1395-1405

[106] Corona BT et al. Further development of a tissue engineered muscle repair construct in vitro for enhanced functional recovery following implantation in vivo in a murine model of volumetric muscle loss injury. Tissue Engineering. Part A. 2012;18(11-12):1213-1228

[107] Wolf MT et al. A hydrogel derived from decellularized dermal extracellular matrix. Biomaterials. 2012;33(29):7028-7038

[108] Goldman SM et al. Co-delivery of micronized urinary bladder matrix damps regenerative capacity of minced muscle grafts in the treatment of volumetric muscle loss injuries. PLoS One. 2017;12(10):e0186593 
[109] Aurora A et al. An acellular biologic scaffold does not regenerate appreciable de novo muscle tissue in rat models of volumetric muscle loss injury. Biomaterials. 2015;67:393-407

[110] Corona BT et al. Implantation of in vitro tissue engineered muscle repair constructs and bladder acellular matrices partially restore in vivo skeletal muscle function in a rat model of volumetric muscle loss injury. Tissue Engineering Part A. 2013;20(3-4):705-715

[111] Badylak S et al. Morphologic study of small intestinal submucosa as a body wall repair device. Journal of Surgical Research. 2002;103(2):190-202

[112] Pollot BE et al. Decellularized extracellular matrix repair of volumetric muscle loss injury impairs adjacent bone healing in a rat model of complex musculoskeletal trauma. Journal of Trauma and Acute Care Surgery. 2016;81(5 Suppl 2 Proceedings of the 2015 Military Health System Research Symposium):S184-S190

[113] Choi JS et al. The influence of electrospun aligned poly(epsilon-caprolactone)/collagen nanofiber meshes on the formation of self-aligned skeletal muscle myotubes. Biomaterials. 2008;29(19):2899-2906

[114] Marcinczyk M et al. Laminin-111 enriched fibrin hydrogels for skeletal muscle regeneration. Biomaterials. 2017;141:233-242

[115] Grefte S et al. Matrigel, but not collagen I, maintains the differentiation capacity of muscle derived cells in vitro. Biomedical Materials. 2012;7(5):055004

[116] Wilschut KJ et al. Extracellular matrix components direct porcine muscle stem cell behavior. Experimental Cell Research. 2010;316(3):341-352

[117] Reing JE et al. Degradation products of extracellular matrix affect cell migration and proliferation. Tissue Engineering. Part A. 2009;15(3):605-614

[118] Vorotnikova E et al. Extracellular matrix-derived products modulate endothelial and progenitor cell migration and proliferation in vitro and stimulate regenerative healing in vivo. Matrix Biology. 2010;29(8):690-700

[119] Brown BN et al. Extracellular matrix as an inductive scaffold for functional tissue reconstruction. Translational Research. 2014;163(4):268-285

[120] Tottey S et al. The effect of source animal age upon extracellular matrix scaffold properties. Biomaterials. 2011;32(1):128-136

[121] Tottey S et al. Extracellular matrix degradation products and low-oxygen conditions enhance the regenerative potential of perivascular stem cells. Tissue Engineering. Part A. $2011 ; 17(1-2): 37-44$

[122] Agrawal V et al. Partial characterization of the Sox2+ cell population in an adult murine model of digit amputation. Tissue Engineering. Part A. 2012;18(13-14):1454-1463 
[123] Turner NJ et al. Xenogeneic extracellular matrix as an inductive scaffold for regeneration of a functioning musculotendinous junction. Tissue Engineering. Part A. 2010;16(11):3309-3317

[124] Crapo PM et al. Effects of biologic scaffolds on human stem cells and implications for CNS tissue engineering. Tissue Engineering. Part A. 2014;20(1-2):313-323

[125] Kasukonis B et al. Codelivery of infusion decellularized skeletal muscle with minced muscle autografts improved recovery from volumetric muscle loss injury in a rat model. Tissue Engineering. Part A. 2016;22(19-20):1151-1163

[126] Mase VJ et al. Clinical application of an acellular biologic scaffold for surgical repair of a large, traumatic quadriceps femoris muscle defect. Orthopedics. 2010;33(7)

[127] Dziki J et al. An acellular biologic scaffold treatment for volumetric muscle loss: Results of a 13-patient cohort study. npj Regenerative Medicine. 2016;1:16008

[128] Aurora A et al. Physical rehabilitation improves muscle function following volumetric muscle loss injury. BMC Sports Science, Medicine and Rehabilitation. 2014;6(1):41 